\title{
Electrocardiograph Lead Placement Location
}

National Cancer Institute

\section{Source}

National Cancer Institute. Electrocardiograph Lead Placement Location. NCI Thesaurus.

Code C87881.

A position, site, or point on the body where electrocardiogram leads are attached. 